\title{
REVIEW
}

\section{Propagation of tau pathology in Alzheimer's disease: identification of novel therapeutic targets}

\author{
Amy M Pooler ${ }^{1,2}$, Manuela Polydoro', Susanne Wegmann'1, Samantha B Nicholls', Tara L Spires-Jones ${ }^{1}$ \\ and Bradley THyman ${ }^{1 *}$
}

\begin{abstract}
Accumulation and aggregation of the microtubule-associated protein tau are a pathological hallmark of neurodegenerative disorders such as Alzheimer's disease (AD). In AD, tau becomes abnormally phosphorylated and forms inclusions throughout the brain, starting in the entorhinal cortex and progressively affecting additional brain regions as the disease progresses. Formation of these inclusions is thought to lead to synapse loss and cell death. Tau is also found in the cerebrospinal fluid (CSF), and elevated levels are a biomarker for AD. Until recently, it was thought that the presence of tau in the CSF was due to the passive release of aggregated tau from dead or dying tangle-bearing neurons. However, accumulating evidence from different AD model systems suggests that tau is actively secreted and transferred between synaptically connected neurons. Transgenic mouse lines with localized expression of aggregating human tau in the entorhinal cortex have demonstrated that, as these animals age, tau becomes mislocalized from axons to cell bodies and dendrites and that human tau-positive aggregates form first in the entorhinal cortex and later in downstream projection targets. Numerous in vitro and in vivo studies have provided insight into the mechanisms by which tau may be released and internalized by neurons and have started to provide insight into how tau pathology may spread in AD. In this review, we discuss the evidence for regulated tau release and its specific uptake by neurons. Furthermore, we identify possible therapeutic targets for preventing the propagation of tau pathology, as inhibition of tau transfer may restrict development of tau tangles in a small subset of neurons affected in early stages of $A D$ and therefore prevent widespread neuron loss and cognitive dysfunction associated with later stages of the disease.
\end{abstract}

\section{Tau pathology in neurodegenerative disease}

Tau is a soluble, unfolded microtubule-associated protein that regulates cytoskeletal dynamics of neurons in the central nervous system [1]. Under pathological conditions, tau becomes abnormally phosphorylated and aggregates into filamentous brain inclusions referred to as neurofibrillary tangles (NFTs) [2]. Although tau composition and structure of NFTs are well characterized, the process of tangle formation is not well understood. The presence of tau inclusions of various morphologies is characteristic of a number of neurodegenerative diseases called tauopathies, including Alzheimer's disease (AD), Pick's disease, and frontotemporal dementia [3]. AD is characterized by neuronal and synapse loss and histopathologically by two hallmark lesions: amyloid- $\beta$-containing plaques and NFTs.

\footnotetext{
* Correspondence: bhyman@partners.org

${ }^{1}$ Massachusetts General Hospital, Harvard Medical School, 114 16th Street, Charlestown, MA 02129, USA

Full list of author information is available at the end of the article
}

In AD, NFTs accumulate in a hierarchical pattern affecting selectively vulnerable brain regions that are essential for learning and memory [4]. Progression of NFT pathology throughout the brain correlates well with decline in cognitive function [5]. The entorhinal cortex (EC) is the first cortical region affected by NFTs, followed by the hippocampus, and ultimately the neocortex [6]. Progression of NFTs appears to propagate across limbic and association cortices, creating a predictable pattern that permits the neuropathological diagnosis of different AD stages [6,7]. The loss of NFT-affected neurons results in disconnection and deafferentation of critical neural circuits [8] and thus is likely to contribute to the defects in memory and higher-order cognitive functions in AD.

The spread of protein aggregates during disease progression is a common theme in many neurodegenerative disorders, including $\alpha$-synuclein in Parkinson's disease [9] and superoxide dismutase-1 in amyotrophic lateral sclerosis 
[10]. However, the mechanism underlying interneuronal spread of these aggregates, including tau, is unknown. Recent studies propose that $\mathrm{AD}$ and other neurodegenerative disorders, though not demonstrated to be infectious, may involve transfer of misfolded proteins between cells, similar to what is observed in prion disease [11,12]. These disorders are said to be 'prion-like', since they lack the infectious properties that characterize true prion diseases (for review, see [13]). However, precisely which cellular mechanisms underlie the spread of protein aggregates, including the propagation of pathological tau in $\mathrm{AD}$, remain unclear.

\section{Models of tau propagation}

Substantial progress has recently been made in understanding the pathogenic mechanisms of tau spreading between neurons and across brain regions. Three independent research groups modeled the process of tau spreading by using transgenic mouse lines with localized expression of aggregating human tau. To generate these mouse lines, neuropsin promoter-driven tTa mice that express this transcriptional activator mainly in the medial EC [14] were crossed with a responder mouse line that expresses human tau carrying the P301L mutation [15]. The resulting mice express transgenic mutant tau almost exclusively in the medial EC [16-18]. As these animals age, the distribution of tau in the brain alters. Tau becomes mislocalized from axons to cell bodies and dendrites in the EC, and human tau-positive NFT-like aggregates form first in the EC and later in downstream projection targets of EC neurons, suggesting transsynaptic propagation of tau [16-18].

Until recently, the spread of tau pathology in AD was attributed to the passive release of aggregated tau from dead or dying tangle-bearing neurons. However, a number of recent studies demonstrated active secretion and interneuronal transfer of tau in different AD model systems [17-25], suggesting that neuronal tau release is a regulated process that could facilitate the trans-neuronal spread of tau misfolding. Therefore, understanding how inter-neuronal transfer of tau occurs may provide potential therapeutic targets to interrupt the proliferation of tau pathology early in disease. In this review, we discuss the cellular mechanisms of tau propagation and potential therapeutic approaches.

\section{Tau is secreted in the absence of neurodegeneration}

Although tau is primarily an axonal cytoplasmic protein, it has also been shown to be present at both the pre- and post-synapse in human brain [26] as well as the postsynaptic compartment of mouse brain [27]. Interestingly, tau directly interacts with synaptic proteins, such as the NMDA receptor [27,28], suggesting a role for tau in regulating intracellular signaling pathways [29]. Extracellularly, tau is also found in brain fluids such as cerebrospinal fluid (CSF) [30] and interstitial fluid (ISF) [31]. Tau is present in the CSF of both healthy and AD individuals, whereby changes in tau concentrations and phosphorylation state are observed in AD and might be useful as a disease biomarker (for review, see [32]). However, the presence of tau in the CSF of healthy individuals, without AD-related widespread neuron loss, is not well understood. Tau is detected in the ISF of awake wild-type mice by using in vivo microdialysis, further suggesting that it is also released by neurons in the absence of neurodegeneration [31]. There is accumulating evidence that tau secretion is an active neuronal process, independent of cell death $[22,23]$, and since tau is present in both axons and dendrites, it is possible that either region may be involved its release. However, tau can also be released from, and taken up by, cultured non-neuronal cells, suggesting that synaptic mechanisms may not be uniquely involved in this process. From cell lines stably expressing tau, it has been estimated that $0.1 \%$ to $0.3 \%$ of total cellular tau is secreted $[33,34]$; however, estimates for neuronal tau secretion in vivo have yet to be determined.

\section{Mechanisms of neuronal tau release}

How do neurons secrete tau? This question has been the subject of several recent studies but remains controversial. Specifically, whether tau is released in a free soluble form or packaged into small membrane vesicles such as exosomes is unclear. Since exosomal membrane is derived from the plasma membrane [35] and tau is associated with the plasma membrane [36,37], it is possible that tau packaged in exosomes is actively secreted in this manner. Consistent with this hypothesis, tau was detected in exosomes isolated from CSF of both healthy, age-matched controls and AD patients [22]. Earlier studies of tau secretion performed in human tau overexpressing non-neuronal cell lines - COS-7 and human embryonic kidney (HEK) - showed that tau release in microvesicles, in the absence of cell death, is an actively regulated process [38]. Furthermore, neuroblastoma M1C cells that inducibly express wild-type human tau secreted both free soluble and exosomal tau [22]. Human tau overexpression in giant neurons of the lamprey caused the release of tau contained within various types of vesicles [20] and subsequent dendritic degeneration. These results suggest that non-microtubule-associated intracellular tau may be packaged and secreted within neuron-derived vesicles. However, whether exosomal tau release is a regulated neuronal process in vivo or results from overexpression and missorting of tau in these cell models remains unclear.

However, a number of recent studies report that tau may be released from cells by a mechanism that does not involve membrane vesicles. Analysis of culture medium 
from HEK cells inducibly expressing human tau, rather than transiently overexpressing tau, revealed soluble extracellular tau but no detectable tau in the exosome fraction [33]. Exosomes isolated from SH-SY5Y neuroblastoma cells, infected with a lentivirus to express human GFPtagged tau, also did not contain tau [39]. Furthermore, increased neuronal activity in cortical neuron cultures stimulates the release of non-exosomal endogenous tau [23]. The absence of tau in neuronal exosomes is supported by proteomic analysis of exosomes derived from cortical neurons [40] and from human neural stem cells [41]. These studies showed that neuron-derived exosomes contain cytoskeletal proteins, including $\beta$-tubulin, but do not possess detectable amounts of tau. Taken together, these studies suggest that free soluble, rather than membrane vesicle-associated, tau is released in the brain. However, under which conditions neurons release tau and whether multiple secretion mechanisms are involved need further investigation.

The discrepancy between the reported exosomal versus soluble secreted tau may result from the use of different model systems: studies showing exosomal or vesicular secretion of tau all involve overexpression of the protein, whereas physiological tau expression levels seem to favor the release of soluble tau. Depending on the cell type, high levels of intracellular tau may lead to toxicity; indeed, exosomal localization of tau was observed in degenerating cells [20]. Furthermore, in a cell culture model, in which tau is inducibly expressed, tau release was detectable in the absence of cytotoxicity and was not associated with vesicles $[21,33]$. In cortical neurons and neuroblastoma cells, the level of tau secretion appears to be low, suggesting a constitutive release of endogenous tau. The amount of tau secreted is greatly increased after stimulation of neuronal activity and seems to depend on changes in intracellular calcium $[23,34]$. Such activity-regulated tau release opens a new view on the progression of tau pathology in $\mathrm{AD}$. There is evidence that disease-relevant tau mutations may have an impact on tau release as well: in vitro expression of tau harboring the P301S mutation in human neuroblastoma cells decreases tau release [34], whereas mice expressing human P301S tau show higher tau levels in ISF compared with wild-type mice [31]. Therefore, further research using animal models of the disease is needed to reveal whether tau release is dynamic and regulated by neuronal activity in vivo and whether it may be altered in neurodegeneration. Importantly, understanding the mechanism of tau secretion in the brain could open the way to new plausible drug targets for the treatment of $\mathrm{AD}$, as reduction of tau secretion may prevent the spread of tangle pathology.

\section{Characterization of extracellular tau}

Since tau has been demonstrated to propagate between neurons, determining which species of tau are involved in its intercellular transfer is of great interest. The phosphorylation state and length of extracellular tau have been reported in numerous studies, but obtaining a global picture is difficult since different epitopes were analyzed in various model systems. Analysis of tau in human CSF found that levels of tau phosphorylated on T181 are increased in patients with AD compared with healthy controls [42]. Phosphorylation of this epitope was also observed in extracellular tau in vitro, released from HEK cells [33] and neuroblastoma M1C cells [22] both inducibly expressing wild-type human tau, and in endogenous tau from SH-SY5Y neuroblastoma cells [43] and mouse cortical neurons [34]. However, tau secreted from cultured cortical neurons was reported to be dephosphorylated at the Tau1 (S199/202) and PHF1 (S396/ 404) epitopes [23]; tau released from transfected HeLa cells was also shown to be dephosphorylated at the AT8 (S202/T205) and PHF1 epitopes [21]. The phosphorylation status of tau as it is released may be difficult to ascertain, however, as extracellular phosphatases may dephosphorylate tau once it is outside the cell [44]. Examination of tau released from human neuroblastoma SH-SY5Y cells revealed that when activity of tissuenonspecific alkaline phosphatase was blocked, levels of extracellular tau phosphorylated at the AT8 and PHF1 epitopes were significantly increased [44]. These results suggest that tau phosphorylation may be altered extracellularly, and this is of particular interest as changes in extracellular tau phosphorylation may underlie tau propagation in $\mathrm{AD}$. Truncation of extracellular tau is the subject of conflicting reports. HeLa cells overexpressing GFP-tagged human tau have been demonstrated to release tau that is cleaved at D421, a caspase-3 cleavage site [21]. However, additional studies report intact, fulllength extracellular tau in vitro [23,34] and in vivo [31].

Although it is known that neurons readily take up tau aggregates $[45,46]$ (as will be discussed in the next section of this review), the extracellular species of tau that propagates between neurons in vivo is not known. Various forms of tau, including monomers, oligomers, or fibrils, could be involved in the spread of tau aggregates. Furthermore, soluble tau monomers or oligomers may be released from cells before tangle formation. A recent study suggests that various types of tau may be transferred between cells, since inoculation of tau-enriched extracts of human brain from individuals with various tauopathies, including $\mathrm{AD}$, frontotemporal dementia, and corticobasoganglionic degeneration, propagated to neurons in a mouse expressing wild-type human tau and even to mice that express only endogenous mouse tau [47]. To date, reports analyzing tau release in cell culture systems [34] or in a mouse model of tauopathy [31] suggest that extracellular tau released from healthy cells is largely monomeric. However, extracellular tau has not 
been characterized in mouse models in which intracerebral inoculation aggregated tau is used to initiate propagation of tangles [46-48]. In these models, released tau may have abnormal phosphorylation or be oligomeric; however, further studies are required to characterize the species of tau that propagates under these conditions.

\section{Neurons internalize extracellular tau}

Knowledge about neuronal uptake of tau, which may facilitate tau spreading in AD and other tauopathies, is still very limited. As discussed above, tau can be released by neurons into extracellular space and therefore may be available for uptake by neighboring cells. Several recent studies have explored the nature of the tau that is taken up by neighboring neurons and aimed to uncover the mechanism by which this process occurs.

Propagation of aggregated tau has been recently explored in vivo by brain inoculation of tau aggregates and monitoring their uptake and propagation in mouse cortex. Injection of tau filaments from transgenic mice expressing human mutant P301S tau into hippocampi of young mice expressing wild-type human tau caused filamentous tau inclusions of the wild-type tau and the spreading of pathology from the site of injection to neighboring brain regions [24]. In a follow-up study, brain extracts from sporadic human tauopathy cases were injected intracerebrally into wild-type mice. Injected mice displayed highly phosphorylated tau inclusions in several brain regions, indicating that, in this model, the presence of mouse tau was sufficient for propagation of pathology [47]. Interestingly, aggregates were found in both astrocytes and neurons [47], suggesting that multiple cell types may be able to internalize tau and participate in propagation of tau pathology.

However, it is not clear which component of the inoculated brain material - the tau aggregates or a soluble tau species - is responsible for transmitting tau pathology. Several studies have directly tested the uptake of various forms of tau to determine whether cells preferentially internalize specific types of tau. For example, in cultured neurons treated with either low-molecularweight aggregates, short fibrils, or long fibrils composed of recombinant full-length tau, only aggregates and short fibrils were internalized [49]. Cells also take up paired helical filaments (PHFs) of tau isolated from AD brain; both HEK and SH-SY5Y neuroblastoma cells internalized exogenously applied PHFs by endocytosis [39]. Furthermore, in C17.2 neural stem cells treated with a monomeric or oligomeric recombinant tau microtubulebinding domain construct (amino acids 243 to 375), a majority of cells took up oligomeric tau, although a small percentage internalized monomeric tau [45]. Interestingly, HEK cells overexpressing this tau construct formed aggregates which were released and internalized by other HEK cells [50], providing direct evidence that microtubule-binding domain fragments of tau aggregates can be transferred between cells in vitro.

Recent in vivo studies have explored whether certain forms of tau are preferentially propagated. To determine whether different types of tau aggregates could be taken up by neurons, synthetic preformed fibrils of recombinant human full-length tau or a truncated form of tau, containing only the microtubule-binding domain, were injected intracerebrally into young mice expressing human mutant P301S tau [46]. Both forms of fibrils were internalized by cells, likely by endocytosis [51], and over time human tau-positive inclusions were found throughout the brain, indicating that both full-length or truncated tau may be propagated in vivo [46]. However, another study, using tau isolated from $A D$ brain, suggests that cells might discriminate in the types of tau internalized in vivo. When oligomeric or PHF tau was injected in the hippocampus of young, wild-type mice, only the tau oligomers, not brain-derived PHF, induced tau pathology 11 months post-injection [48]. Interestingly, both oligomeric and PHF tau were internalized by cells near the injection site, but only oligomeric tau was propagated to other brain regions, suggesting that PHF tau may not be transported and released by neurons [48]. This study supports a critical role of mouse tau in propagation of tau pathology in mouse models since, in tau knockout mice, no propagation of oligomeric tau was observed. Furthermore, the aggregates found in brain areas distal to the injection site were composed of mouse and not human tau, indicating that the tau propagation was not simply transfer of the injected material [48], in agreement with recently reported findings using a different mouse model [47].

The exact mechanism by which cells internalize tau remains unknown; it may be dependent upon the tau species (for example, monomers, small soluble oligomers, or aggregates). Further work is required to better understand whether the spread of tau inclusions is due to a prion-like mechanism, in which misfolded tau induces other tau molecules to become similarly misfolded [52]. In this manner, misfolded or aggregated tau might act as a template, altering the conformation of tau in each neuron that internalizes it. However, in the case of tau aggregates, it remains unclear whether misfolded tau can alter conformation of other tau molecules directly or whether these aggregates interact with other cellular factors, like kinases, to alter tau in a way that would induce changes in conformation [53]. Internalization of tau may be different for isoforms that vary in number of microtubule repeat domains and $\mathrm{N}$-terminal inserts or in post-translational modifications such as phosphorylation and truncation state. The studies discussed above indicate that soluble oligomeric tau is taken up and released 
both in vivo [48] and in vitro [45] and that this species facilitates the transmission of tau misfolding between cells, suggesting that therapies such as small-molecule tau aggregation inhibitors (reviewed in [54]), which might reduce accumulation of extracellular tau oligomers, might also slow transmission of tau pathology.

\section{Therapeutic intervention and tau propagation}

Accumulating evidence therefore suggests that inhibiting the intercellular transfer of tau may slow or halt the progression of tau pathology in AD. Furthermore, as tau pathology is associated with synapse loss [55] and subsequent cell death [56], prevention of its spread may also impact disease-related cognitive decline. Therapeutic strategies for preventing or reducing the interneuronal transfer of tau may take several approaches: blocking tau release, reducing accumulation or oligomerization of extracellular tau, or preventing tau uptake (Figure 1). Targeting tau release may enable reduction of extracellular tau levels, thereby reducing its availability for uptake by neighboring neurons. Since neurons release tau in vivo [31], possibly in an activity-dependent manner [23], further research is needed to determine the precise mechanism, which would be crucial for identifying targets for therapeutic intervention. Interestingly, the presence of extracellular tau in the absence of neurodegeneration, as described above, suggests that perhaps tau may have a yet unknown function outside the cell (for example, by participating in cell-cell signaling). Indeed, exogenously applied recombinant tau has been shown to bind and activate muscarinic acetylcholine receptors in cultured hippocampal neurons with an affinity higher than that of acetylcholine $[57,58]$. Additional work is needed to determine whether tau can stimulate these receptors in vivo, as these studies suggest that, although inhibition of tau release may halt inter-neuronal transfer of tau pathology, care must be taken to ensure that normal functions of extracellular tau are preserved. Furthermore, although recent studies suggest that neurons may preferentially internalize and propagate oligomeric tau in vivo [47,48],

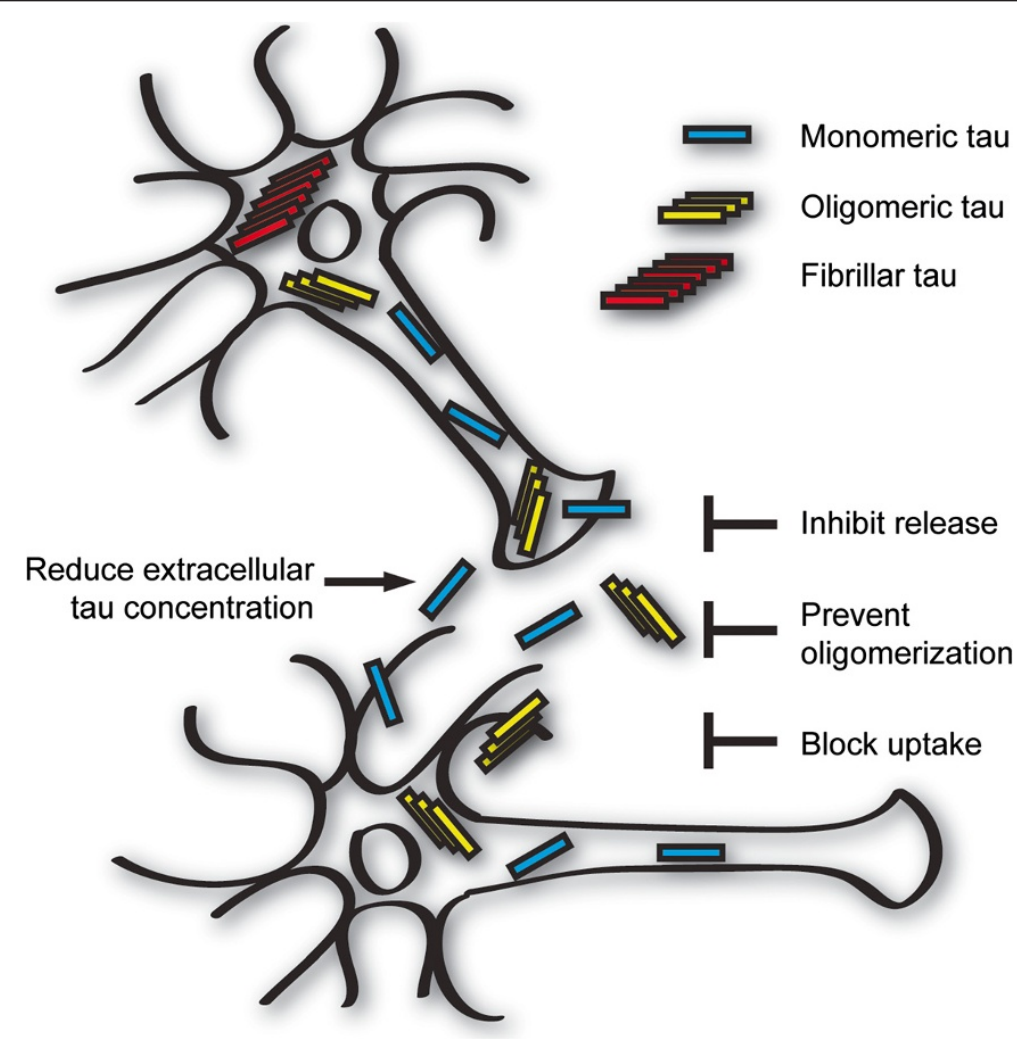

Figure 1 Potential therapeutic targets to slow or prevent the spread of tau pathology in the brain. The mechanisms by which toxic tau species are transferred between cells are not known; further investigation is required to understand how tau is released into extracellular space and internalized. It is also unclear which species of tau are involved at each stage; neurons may release monomeric or oligomeric tau, which may include toxic forms of soluble tau. Released tau may also be misfolded, and may have modifications such as abnormal phosphorylation, truncation or both. As these mechanisms are better understood, potential therapeutic targets that will prevent or slow the spread of pathological tau may be uncovered. For example, the release and uptake of tau may be inhibited pharmacologically, and immunotherapy might lower extracellular tau concentration. Furthermore, anti-aggregant drugs may prevent tau oligomerization, reducing levels of potentially toxic forms of tau that are available to be internalized. 
mechanisms of uptake of extracellular tau also require additional research in order to identify which type (or types) of tau may be internalized and how this process may occur.

The spread of pathogenic tau may also be slowed or prevented by treatments that reduce levels of extracellular tau or that alter tau such that neurons are no longer able to internalize it. For example, inhibition or reversion of extracellular tau oligomerization might interfere with tau pathology spreading [54]; however, care needs to be taken with this strategy, as disruption of tau aggregates may result in formation of other soluble species of tau, which may also be toxic [59]. Therefore, prevention of extracellular tau accumulation or removal of pathological tau species may prove to be a more cautious method. Interestingly, recent studies of passive immunization of a tauopathy mouse model with antibodies directed against tau significantly prevented the formation of NFTs [60] or ameliorated existing tau pathology in older animals [61]. Active immunization, in which mice were inoculated with tau peptides, also proved effective at preventing and improving tau pathology in mice overexpressing human P301L tau [62]. Although tau immunotherapy has been successful in animal models, clinical trials will provide important information about whether anti-tau immunotherapies may slow or reverse disease progression in AD [63]. These trials may demonstrate a reduction in tau inclusions in AD brain, as found in an animal model of disease [61]. Furthermore, inhibition of tau transfer may restrict development of tau tangles to brain regions affected in early stages of $\mathrm{AD}$ and therefore prevent the cognitive dysfunction associated with later stages of the disease.

\section{Conclusions}

Propagation of tau pathology in the brain is a hallmark of AD. However, only recently have the mechanisms underlying the interneuronal transmission of tau been explored. Further understanding of these processes will be important for identification of novel therapeutic targets aimed at halting the spread of tau aggregation. Although progress has been made in identifying the tau species involved in propagation, further research is needed both in vitro and in vivo in order to identify precisely which forms of tau are involved in transmission of pathology. Identifying these pathological tau species is particularly important therapeutically, since these transmissible forms of tau may represent a specific target for immunotherapy. Crucially, it will be important to target pathological tau to minimize interference with normal tau function.

Numerous questions with regard to how tau pathology might be propagated in disease remain. For example, further research may provide insight into whether nonneuronal cell types regulate intercellular tau transfer, since glia are able to internalize tau aggregates in a mouse model of tauopathy [47] and glial tangles are also found in frontotemporal dementia [64]. Furthermore, although tau pathology appears to propagate along neural networks $[17,18]$ and its release is regulated by synaptic activity [23], whether propagation of pathology occurs at synapses remains to be demonstrated [26]. Therefore, further identification and characterization of mechanisms of tau release and uptake will be critical for therapeutic interventions that may prevent or slow neurodegeneration in AD.

\section{Note: This article is part of a series on Tau-based therapeutic strategies, edited by Leonard Petrucelli. Other articles in this series can be found at http://alzres.com/series/tau_therapeutics.}

\section{Abbreviations}

AD: Alzheimer's disease; CSF: Cerebrospinal fluid; EC: Entorhinal cortex; HEK: Human embryonic kidney; ISF: Interstitial fluid; NFT: Neurofibrillary tangle; PHF: Paired helical filament.

\section{Competing interests}

The authors declare that they have no competing interests.

\section{Acknowledgments}

This work was supported by R00AG033670, R01AG026249-07, 5T32AG00022222, and R21 NS067127 and grants from the American Health Assistance Foundation, Alzheimer's Research UK, and the Deutsche Forschungsgemeinschaft (DFG).

\section{Author details}

${ }^{1}$ Massachusetts General Hospital, Harvard Medical School, 114 16th Street, Charlestown, MA 02129, USA. 'Institute of Psychiatry, Department of Neuroscience, King's College London, DeCrespigny Park, London SE5 8AF, UK.

\section{Published: 23 Oct 2013}

\section{References}

1. Trinczek B, Biernat J, Baumann K, Mandelkow EM, Mandelkow E: Domains of tau protein, differential phosphorylation, and dynamic instability of microtubules. Mol Biol Cell 1995, 6:1887-1902.

2. Duyckaerts C, Delatour B, Potier MC: Classification and basic pathology of Alzheimer disease. Acta Neuropathol 2009, 118:5-36.

3. Lee G, Leugers CJ: Tau and tauopathies. Prog Mol Biol Trans/ Sci 2012, 107:263-293.

4. Hyman BT, Van Hoesen GW, Damasio AR, Barnes CL: Alzheimer's disease: cell-specific pathology isolates the hippocampal formation. Science 1984, 225:1168-1170.

5. Nelson PT, Alafuzoff I, Bigio EH, Bouras C, Braak H, Cairns NJ, Castellani RJ, Crain BJ, Davies P, Del Tredici K, Duyckaerts C, Frosch MP, Haroutunian V, Hof PR, Hulette CM, Hyman BT, Iwatsubo T, Jellinger KA, Jicha GA, Kövari E, Kukull WA, Leverenz JB, Love S, Mackenzie IR, Mann DM, Masliah E, McKee AC Montine TJ, Morris JC, Schneider JA, Sonnen JA, Thal DR, Trojanowski JQ, Troncoso JC, Wisniewski T, Woltjer RL, Beach TG: Correlation of Alzheimer disease neuropathologic changes with cognitive status: a review of the literature. J Neuropathol Exp Neuro/ 2012, 71:362-381.

6. Braak H, Braak E: Neuropathological stageing of Alzheimer-related changes. Acta Neuropathol 1991, 82:239-259.

7. Arnold SE, Hyman BT, Flory J, Damasio AR, Van Hoesen GW: The topographical and neuroanatomical distribution of neurofibrillary tangles and neuritic plaques in the cerebral cortex of patients with Alzheimer's disease. Cereb Cortex 1991, 1:103-116.

8. Hyman BT, Van Hoesen GW, Damasio AR: Memory-related neural systems in Alzheimer's disease: an anatomic study. Neurology 1990, 40:1721-1730. 
9. Desplats P, Lee HJ, Bae EJ, Patrick C, Rockenstein E, Crews L, Spencer B, Masliah E, Lee SJ: Inclusion formation and neuronal cell death through neuron-to-neuron transmission of alpha-synuclein. Proc Natl Acad Sci USA 2009, 106:13010-13015.

10. Munch C, O'Brien J, Bertolotti A: Prion-like propagation of mutant superoxide dismutase-1 misfolding in neuronal cells. Proc Natl Acad Sci USA 2011, 108:3548-3553.

11. Holmes BB, Diamond MI: Cellular mechanisms of protein aggregate propagation. Curr Opin Neurol 2012, 25:721-726.

12. Hofmann JP, Denner $P$, Nussbaum-Krammer $C$, Kuhn $\mathrm{PH}$, Suhre $M \mathrm{H}_{\text {, }}$ Scheibel T, Lichtenthaler SF, SchatzI HM, Bano D, Vorberg IM: Cell-to-cell propagation of infectious cytosolic protein aggregates. Proc Natl Acad Sci USA 2013, 110:5951-5956.

13. Kaufman SK, Diamond MI: Prion-like propagation of protein aggregation and related therapeutic strategies. Neurotherapeutics 2013, 10:371-382

14. Yasuda M, Mayford MR: CaMKII activation in the entorhinal cortex disrupts previously encoded spatial memory. Neuron 2006, 50:309-318.

15. Santacruz K, Lewis J, Spires T, Paulson J, Kotilinek L, Ingelsson M, Guimaraes A, DeTure M, Ramsden M, McGowan E, Forster C, Yue M, Orne J, Janus C, Mariash A, Kuskowski M, Hyman B, Hutton M, Ashe KH: Tau suppression in a neurodegenerative mouse model improves memory function. Science 2005, 309:476-481.

16. Harris JA, Koyama A, Maeda S, Ho K, Devidze N, Dubal DB, Yu GQ, Masliah E, Mucke L: Human P301L-mutant tau expression in mouse entorhinalhippocampal network causes tau aggregation and presynaptic pathology but no cognitive deficits. PLoS One 2012, 7:e45881.

17. de Calignon A, Polydoro M, Suárez-Calvet M, William C, Adamowicz DH, Kopeikina KJ, Pitstick R, Sahara N, Ashe KH, Carlson GA, Spires-Jones TL, Hyman BT: Propagation of tau pathology in a model of early Alzheimer's disease. Neuron 2012, 73:685-697.

18. Liu L, Drouet V, Wu JW, Witter MP, Small SA, Clelland C, Duff K: Trans-synaptic spread of tau pathology in vivo. PLoS One 2012, 7:e31302.

19. Kim W, Lee S, Jung C, Ahmed A, Lee G, Hall GF: Interneuronal transfer of human tau between Lamprey central neurons in situ. J Alzheimers Dis 2010, 19:647-664.

20. Lee S, Kim W, Li Z, Hall GF: Accumulation of vesicle-associated human tau in distal dendrites drives degeneration and tau secretion in an in situ cellular tauopathy model. Int J Alzheimers Dis 2012, 2012:172837.

21. Plouffe V, Mohamed NV, Rivest-McGraw J, Bertrand J, Lauzon M, Leclerc N: Hyperphosphorylation and cleavage at D421 enhance tau secretion. PLoS One 2012, 7:e36873

22. Saman S, Kim W, Raya M, Visnick Y, Miro S, Jackson B, McKee AC, Alvarez VE, Lee NC, Hall GF: Exosome-associated tau is secreted in tauopathy models and is selectively phosphorylated in cerebrospinal fluid in early Alzheimer disease. J Biol Chem 2012, 287:3842-3849.

23. Pooler AM, Phillips EC, Lau DH, Noble W, Hanger DP: Physiological release of endogenous tau is stimulated by neuronal activity. EMBO Rep 2013, 14:389-394.

24. Clavaguera F, Bolmont T, Crowther RA, Abramowski D, Frank S, Probst A, Fraser G, Stalder AK, Beibel M, Staufenbiel M, Jucker M, Goedert M, Tolnay M: Transmission and spreading of tauopathy in transgenic mouse brain. Nat Cell Biol 2009, 11:909-913.

25. Le MN, Kim W, Lee S, McKee AC, Hall GF: Multiple mechanisms of extracellular tau spreading in a non-transgenic tauopathy model. Am J Neurodegener Dis 2012, 1:316-333.

26. Tai HC, Serrano-Pozo A, Hashimoto T, Frosch MP, Spires-Jones $T L$, Hyman BT: The synaptic accumulation of hyperphosphorylated tau oligomers in Alzheimer disease is associated with dysfunction of the ubiquitin-proteasome system. Am J Pathol 2012, 181:1426-1435.

27. Ittner LM, Ke YD, Delerue F, Bi M, Gladbach A, van Eersel J, Wölfing H, Chieng BC, Christie MJ, Napier IA, Eckert A, Staufenbiel M, Hardeman E, Götz J: Dendritic function of tau mediates amyloid-beta toxicity in Alzheimer's disease mouse models. Cell 2010, 142:387-397.

28. Mondragon-Rodriquez S, Trillaud-Doppia E, Dudilot A, Bourgeois C, Lauzon M Leclerc N, Boehm J: Interaction of endogenous tau protein with synaptic proteins is regulated by $\mathrm{N}$-methyl-D-aspartate receptor-dependent tau phosphorylation. J Biol Chem 2012, 287:32040-32053.

29. Pooler AM, Hanger DP: Functional implications of the association of tau with the plasma membrane. Biochem Soc Trans 2010, 38:1012-1015.

30. Arai H, Terajima M, Miura M, Higuchi S, Muramatsu T, Machida N, Seiki H, Takase S, Clark CM, Lee VM, Trojanowski JQ, Sasaki H: Tau in cerebrospinal fluid: a potential diagnostic marker in Alzheimer's disease. Ann Neurol 1995, 38:649-652.

31. Yamada K, Cirrito JR, Stewart FR, Jiang H, Finn MB, Holmes BB, Binder LI, Mandelkow EM, Diamond MI, Lee VM, Holtzman DM: In vivo microdialysis reveals age-dependent decrease of brain interstitial fluid tau levels in P301S human tau transgenic mice. J Neurosci 2011, 31:13110-13117.

32. Kang JH, Korecka M, Toledo JB, Trojanowski JQ, Shaw LM: Clinical utility and analytical challenges in measurement of cerebrospinal fluid amyloid-beta1-42 and tau proteins as Alzheimer disease biomarkers. Clin Chem 2013, 59:903-916.

33. Chai $X$, Dage $J$, Citron M: Constitutive secretion of tau protein by an unconventional mechanism. Neurobiol Dis 2012, 48:356-366.

34. Karch CM, Jeng AT, Goate AM: Extracellular Tau levels are influenced by variability in Tau that is associated with tauopathies. J Biol Chem 2012, 287:42751-42762

35. Lopez-Verrilli MA, Court FA: Exosomes: mediators of communication in eukaryotes. Biol Res 2013, 46:5-11.

36. Brandt R, Leger J, Lee G: Interaction of tau with the neural plasma membrane mediated by tau's amino-terminal projection domain. J Cell Biol 1995, 131:1327-1340.

37. Pooler AM, Usardi A, Evans CJ, Philpott KL, Noble W, Hanger DP: Dynamic association of tau with neuronal membranes is regulated by phosphorylation. Neurobiol Aging 2012, 33:431-438. e427-438.

38. Simon D, Garcia-Garcia E, Royo F, Falcon-Perez JM, Avila J: Proteostasis of tau. Tau overexpression results in its secretion via membrane vesicles. FEBS Lett 2012, 586:47-54.

39. Santa-Maria I, Varghese M, Ksiezak-Reding H, Dzhun A, Wang J, Pasinetti GM Paired helical filaments from Alzheimer disease brain induce intracellular accumulation of Tau protein in aggresomes. J Biol Chem 2012, 287:20522-20533.

40. Fauré J, Lachenal G, Court M, Hirrlinger J, Chatellard-Causse C, Blot B, Grange J, Schoehn G, Goldberg Y, Boyer V, Kirchhoff F, Raposo G, Garin J, Sadoul R: Exosomes are released by cultured cortical neurones. Mol Cell Neurosci 2006, 31:642-648.

41. Kang D, Oh S, Ahn SM, Lee BH, Moon MH: Proteomic analysis of exosomes from human neural stem cells by flow field-flow fractionation and nanoflow liquid chromatography-tandem mass spectrometry. J Proteome Res 2008, 7:3475-3480

42. Vanmechelen $E$, Vanderstichele $H$, Davidsson $P$, Van Kerschaver $E$, Van Der Perre B, Sjogren M, Andreasen N, Blennow K: Quantification of tau phosphorylated at threonine 181 in human cerebrospinal fluid: a sandwich ELISA with a synthetic phosphopeptide for standardization. Neurosci Lett 2000, 285:49-52.

43. Karch CM, Jeng AT, Goate AM: Calcium phosphatase calcineurin influences tau metabolism. Neurobiol Aging 2013, 34:374-386.

44. Diaz-Hernandez M, Gomez-Ramos A, Rubio A, Gomez-Villafuertes R, Naranjo JR, Miras-Portugal MT, Avila J: Tissue-nonspecific alkaline phosphatase promotes the neurotoxicity effect of extracellular tau. J Biol Chem 2010, 285:32539-32548.

45. Frost B, Jacks RL, Diamond Ml: Propagation of tau misfolding from the outside to the inside of a cell. J Biol Chem 2009, 284:12845-12852.

46. Iba M, Guo JL, McBride JD, Zhang B, Trojanowski JQ, Lee VM: Synthetic tau fibrils mediate transmission of neurofibrillary tangles in a transgenic mouse model of Alzheimer's-like tauopathy. J Neurosci 2013, 33:1024-1037.

47. Clavaquera F, Akatsu H, Fraser G, Crowther RA, Frank S, Hench J, Probst A, Winkler DT, Reichwald J, Staufenbiel M, Ghetti B, Goedert M, Tolnay M: Brain homogenates from human tauopathies induce tau inclusions in mouse brain. Proc Natl Acad Sci U S A 2013, 110:9535-9540.

48. Lasagna-Reeves CA, Castillo-Carranza DL, Sengupta U, Guerrero-Munoz MJ, Kiritoshi T, Neugebauer V, Jackson GR, Kayed R: Alzheimer brain-derived tau oligomers propagate pathology from endogenous tau. Sci Rep 2012, 2:700

49. Wu JW, Herman M, Liu L, Simoes S, Acker CM, Figueroa H, Steinberg J, Margittai M, Kayed R, Zurzolo C, Di Paolo G, Duff KE: Small misfolded Tau species are internalized via bulk endocytosis and anterogradely and retrogradely transported in neurons. J Biol Chem 2013, 288:1856-1870.

50. Kfoury N, Holmes BB, Jiang H, Holtzman DM, Diamond Ml: Trans-cellular propagation of Tau aggregation by fibrillar species. J Biol Chem 2012, 287:19440-19451.

51. Guo JL, Lee VM: Seeding of normal Tau by pathological Tau conformers drives pathogenesis of Alzheimer-like tangles. J Biol Chem 2011, 286:15317-15331. 
52. Frost B, Diamond MI: Prion-like mechanisms in neurodegenerative diseases. Nat Rev Neurosci 2010, 11:155-159.

53. Walker LC, Diamond MI, Duff KE, Hyman BT: Mechanisms of protein seeding in neurodegenerative diseases. JAMA Neurol 2013, 70:304-310.

54. Bulic B, Pickhardt M, Mandelkow E: Progress and developments in tau aggregation inhibitors for Alzheimer disease. J Med Chem 2013, 56:4135-4155,

55. Kopeikina KJ, Polydoro M, Tai HC, Yaeger E, Carlson GA, Pitstick R, Hyman BT, Spires-Jones TL: Synaptic alterations in the rTg4510 mouse model of tauopathy. J Comp Neurol 2013, 521:1334-1353.

56. de Calignon A, Fox LM, Pitstick R, Carlson GA, Bacskai BJ, Spires-Jones TL, Hyman BT: Caspase activation precedes and leads to tangles. Nature 2010, 464:1201-1204.

57. Gomez-Ramos A, Diaz-Hernandez M, Rubio A, Diaz-Hernandez Jl, Miras-Portugal MT, Avila J: Characteristics and consequences of muscarinic receptor activation by tau protein. Eur Neuropsychopharmacol 2009, 19:708-717.

58. Gomez-Ramos A, Diaz-Hernandez M, Rubio A, Miras-Portugal MT, Avila J: Extracellular tau promotes intracellular calcium increase through M1 and M3 muscarinic receptors in neuronal cells. Mol Cell Neurosci 2008, 37:673-681.

59. Crimins JL, Pooler A, Polydoro M, Luebke Jl, Spires-Jones TL: The intersection of amyloid beta and tau in glutamatergic synaptic dysfunction and collapse in Alzheimer's disease. Ageing Res Rev 2013, 12:757-763.

60. Chai X, Wu S, Murray TK, Kinley R, Cella CV, Sims H, Buckner N, Hanmer J, Davies P, O'Neill MJ, Hutton ML, Citron M: Passive immunization with anti-Tau antibodies in two transgenic models: reduction of Tau pathology and delay of disease progression. J Biol Chem 2011, 286:34457-34467.

61. Boutajangout A, Ingadottir J, Davies P, Sigurdsson EM: Passive immunization targeting pathological phospho-tau protein in a mouse model reduces functional decline and clears tau aggregates from the brain. J Neurochem 2011, 118:658-667.

62. Bi M, Ittner A, Ke YD, Gotz J, Ittner LM: Tau-targeted immunization impedes progression of neurofibrillary histopathology in aged P301L tau transgenic mice. PLoS One 2011, 6:e26860.

63. Yu YJ, Watts RJ: Developing therapeutic antibodies for neurodegenerative disease. Neurotherapeutics 2013, 10:459-472.

64. Taniguchi S, McDonagh AM, Pickering-Brown SM, Umeda Y, Iwatsubo T, Hasegawa M, Mann DM: The neuropathology of frontotemporal lobar degeneration with respect to the cytological and biochemical characteristics of tau protein. Neuropathol Appl Neurobiol 2004, 30:1-18.

\subsection{6/alzrt214}

Cite this article as: Pooler et al:: Propagation of tau pathology in Alzheimer's disease: identification of novel therapeutic targets. Alzheimer's Research \& Therapy 2013, 5:49 